\title{
Radio Observations of High Energy Solar Flares
}

\author{
Mukul R. Kundu \\ Department of Astronomy, University of Maryland, College Park, MD \\ 20742, USA
}

\begin{abstract}
In this paper we discuss some recent observations of radio emission produced by high energy electrons resulting from solar flares. We discuss the spatial structure of simple weak spiky burst emissions observed simultaneously, at 17 and $34 \mathrm{GHz}$ with the Nobeyama Radio Heliograph (NoRH). Using simultaneous imaging observations obtained by Yohkoh/HXT and SXT along with NoRH 17 \& $34 \mathrm{GHz}$ observations, we attempt to model a relatively stronger and longer flare using gyrosynchrotron mechanism for the flaring radio emission.
\end{abstract}

\section{Introduction}

Radio observations of flares can play a major role in our understanding of their properties. Flares produce strong emission from electrons accelerated to energies in excess of $20 \mathrm{keV}$ via the gyrosynchrotron process: thus radio observations are capable of imaging these electrons directly in the solar corona. The only other technique by which such electrons can be imaged is with hard X-ray telescopes, and because the bremsstrahlung process which produces hard X-ray photons has properties quite different from gyrosynchrotron emission, quite complementary information is obtained from the two techniques. Combination of imaging data at these wavelengths provides one of the most powerful methods available for studying solar flares. In this paper we present some results on flares obtained with the 17 and $34 \mathrm{GHz}$ imaging data acquired by $\mathrm{NoRH}$, along with Yohkoh HXT and SXT imaging data, in order to address the origin of solar flares, particle acceleration and energy transport problems.

\section{Observations and Results}

\subsection{Spatial Structure of Simple Spiky Bursts at Microwave/Milli- meter Wavelengths}

We have investigated the spatial structure of sources of microwave and millimeter bursts with simple spiky time profiles at 17 and $34 \mathrm{GHz}$, similar to those found to be common at $3 \mathrm{~mm}$ wavelength (White 1994). These bursts of short duration exhibit a linear rise time of less than $10 \mathrm{~s}$ and an exponential decay of time constant less than $15 \mathrm{~s}$, which is similar to a time profile shape found to be common at $3 \mathrm{~mm}$ wavelength. These bursts can be of any intensity, from $\sim 1$ sfu to 10 s of $s f u$; they are often strongly polarized $(z 50 \%)$. When mapped 


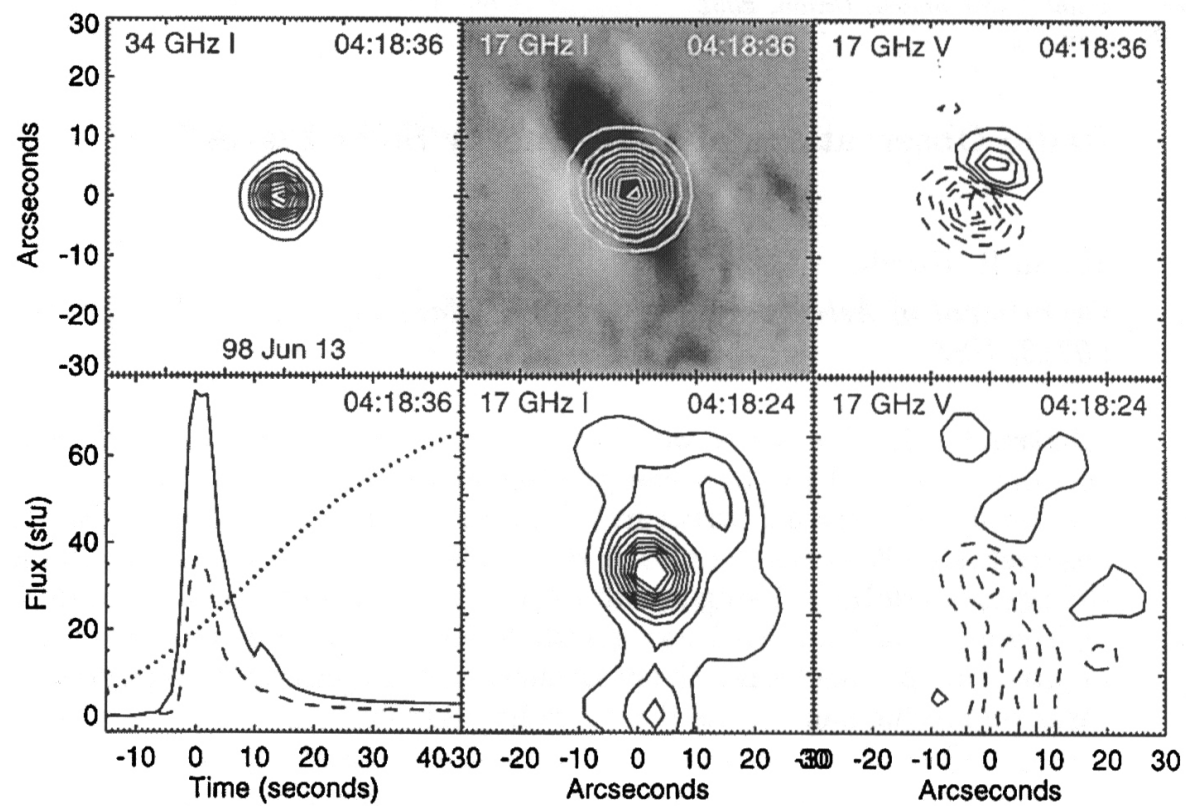

Figure 1. NoRH time profiles and images of the flare at 04:18:36 UT on 1998 June 13 . The three upper panels show images at the peak of the flare at $34 \mathrm{GHz}$ in total intensity in the left panel, at $17 \mathrm{GHz}$ in total intensity and circular polarization in the middle and the right panel respectively. The grey-scale image in the upper middle panel is a SOHO/MDI longitudinal magnetogram taken at 01:35:04 UT, with a linear display of magnetic field strength saturating at $\pm 400 \mathrm{G}$. The lower left panel shows 1 second resolution time profiles of the radio flux at $17 \mathrm{GHz}$ (solid line) and $34 \mathrm{GHz}$ (dashed line) and a 3 second resolution profile of the soft X-ray flux measured by the GOES satellite in the $1.5-10 \mathrm{keV}$ channel (dotted line, scaled arbitrarily). The lower middle and right panels show a pre-flare image in total intensity and circular polarization at $17 \mathrm{GHz}$.

at high spatial resolution with the Nobeyama Radio Heliograph (NoRH), direct evidence that the radio sources are compact bipolar loops is seen (Figure 1): three of the five events studied by Kundu et al. (2000b) show bipolar sources in the circular polarization maps. We interpret these as the oppositely polarized footpoints of loops whose size is less than 5". Every one of the events lies over a neutral line in the photospheric magnetogram and the bipolar $\mathrm{V}$ sources have orientations consistent with the footpoints of a loop straddling the neutral line. Such small source sizes are consistent with the short durations of the events. We interpret them as simple single-loop flares. The soft X-ray behavior is not entirely consistent with the Neupert effect in these events. 


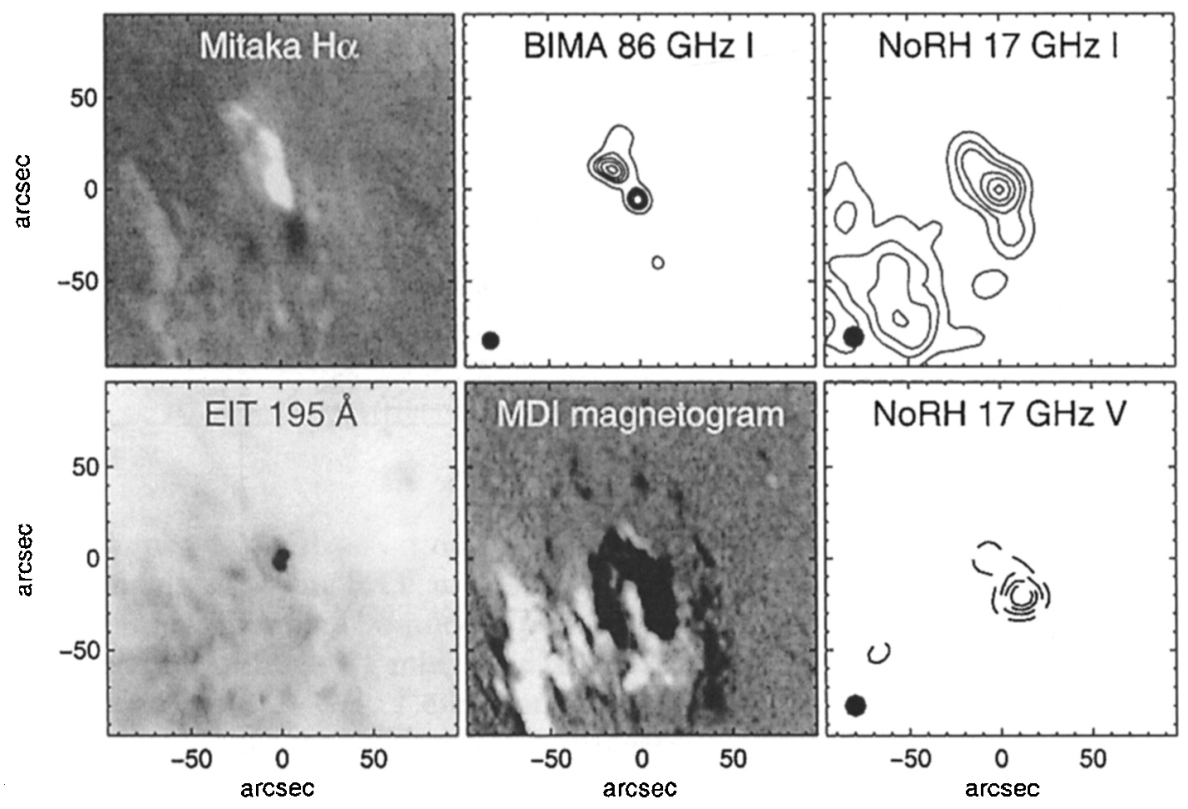

Figure 2. Images of the 1998 Nov. 24 flare at a number of wavelengths. The top left panel shows a line-center $\mathrm{H} \alpha$ image of the flare, and of the sunspot just south-west of the flare site, taken by by the Solar Flare Telescope at Mitaka, Japan, at 23:41:52 UT. The upper middle panel shows total intensity contours at $86 \mathrm{GHz}$ from BIMA at 23:41:20, and the two right-hand panels show NoRH $17 \mathrm{GHz}$ images in total intensity (upper) and circularly-polarized flux (lower panel) at 23:41:10 UT. The bottom left panel shows an EIT $195 \AA$ image obtained at 23:48 UT, and the lower middle panel shows an MDI magnetogram obtained at 22:24 UT with the display clipped at $\pm 150 \mathrm{G}$.

\subsection{Non-thermal Flare Emission From MeV-Energy Electrons at 17, 34 and $86 \mathrm{GHz}$}

It has been shown (White 1994; Kundu et al. 1994) that in solar flares the radio emission at millimeter wavelengths is most sensitive to electrons with energies in excess of $1 \mathrm{MeV}$, i.e., those electrons whose bremsstrahlung should be in the gamma ray regime rather than those which emit hard X-rays in the range 25 - $1000 \mathrm{keV}$. Observations with a millimeter interferometer are very sensitive, and can thus test whether or not "typical" solar flares produce MeV-energy electrons. The BIMA observations indicate that most small impulsive flares do indeed produce MeV-energy electrons (Kundu et al. 1994; White 1994), and hence the required acceleration mechanism can operate even in conditions of relatively weak energy releases.

We have analyzed two solar flares observed with high spatial resolution at $86 \mathrm{GHz}$ ( $3 \mathrm{~mm}$ wavelength) with the BIMA millimeter-wavelength telescope and at 17 and $34 \mathrm{GHz}$ with the Nobeyama Radioheliograph. One of the two events we present is the first clear case of $\lambda=3 \mathrm{~mm}$ source in which both footpoints 


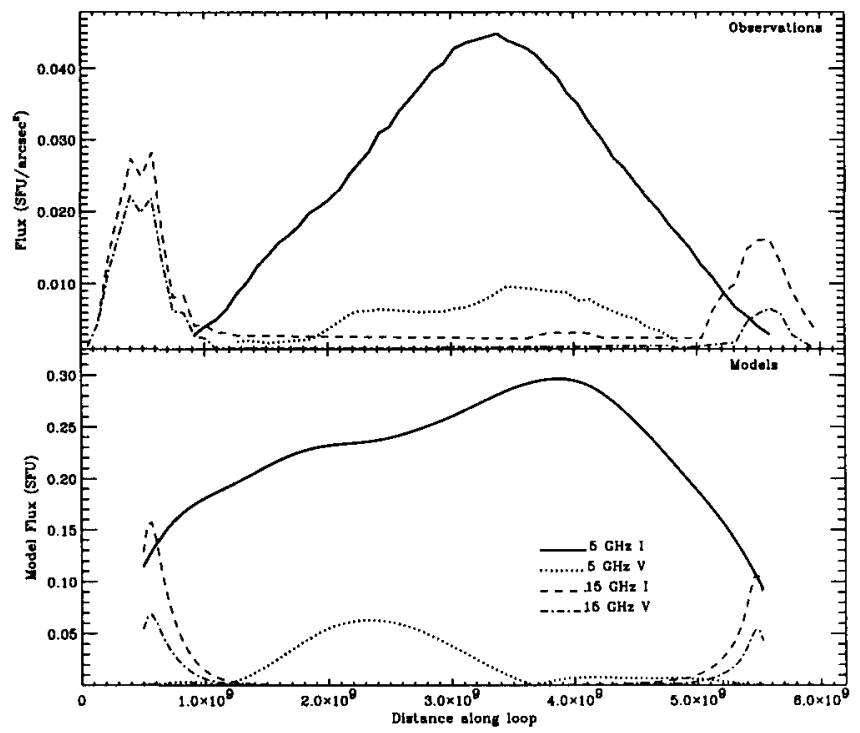

Figure 3. Top panel: One-dimensional representation of the intensity of the VLA flare on 1992 July 1 at the time of maximum. These profiles were computed along the "spine" of the flaring loop. Bottom panel: spatial profiles of the models used to fit the flare as a function of the distance along the loop.

of a loop are detected (Figure 2). In the second event the polarization image at $17 \mathrm{GHz}$ also suggests a bipolar loop-like morphology. Such morphological observations can be used to constrain the nature of the magnetic field in the solar corona (Kundu et al. 2000a).

\subsection{Microwave Observations of Flaring Loops and Flare Modeling}

We have used simultaneous images of flaring loops at two microwave frequencies (5 and $15 \mathrm{GHz}$ ) to model the magnetic structure of a flaring loop and the energy distribution of the radiating electrons. At $15 \mathrm{GHz}$, the flare emission was optically thin and came from the footpoints of the flaring loop, while at $5 \mathrm{GHz}$ the loop itself was outlined. Most of the $5 \mathrm{GHz}$ emission was optically thick, and its spatial maximum was close to the loop top. We compared the observations with calculations of gyrosynchrotron emission from an inhomogeneous magnetic loop (Figure 3) in order to determine the conditions in the flaring loop (Nindos et al. 2000). The best fit to the observations was reached with a model flaring loop with photospheric footpoint magnetic field strength of $870 \mathrm{G}$. The thickness of the model loop was small compared with its footpoint separation. The energy spectral index of the energetic electrons was 3.7 , and their number density was $7.9 \times 10^{7} \mathrm{~cm}^{-3}$. The low- and high-energy cutoffs of the non-thermal electrons were 8 and $210 \mathrm{keV}$, respectively.

We have modeled several relatively strong and longer lasting (than simple spiky events) events at 17 and $34 \mathrm{GHz}$ along with $\mathrm{HXT}$ imaging data. The NoRH 


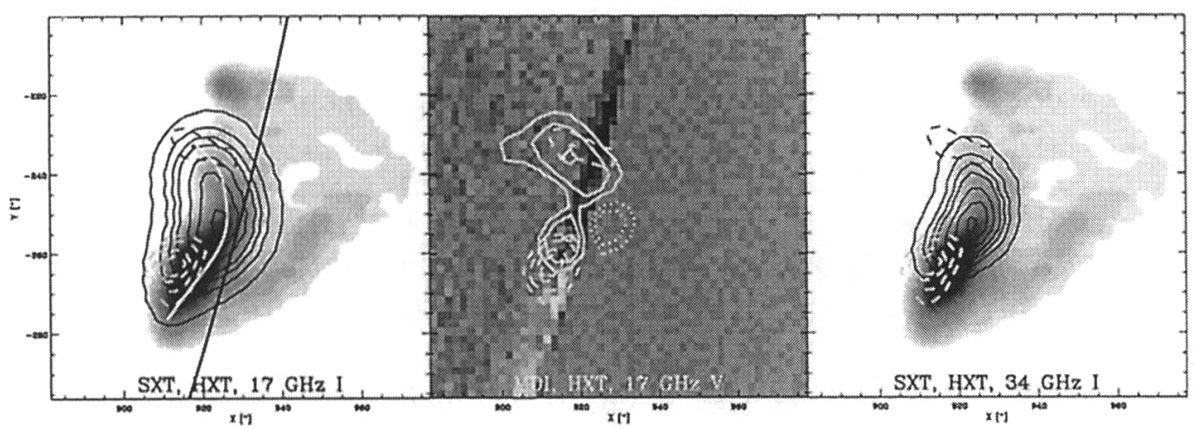

Figure 4. Observations of the 1998 May 8 flare. The left and right panels show an SXT image obtained $10 \mathrm{~s}$ before the time of the radio maximum. The middle panel shows an MDI photospheric magnetogram obtained 1 min after the flare. The black solid contours show the 17 and $34 \mathrm{GHz} I$ emission at the time of maximum. In the middle panel the white solid and dotted contours show the $17 \mathrm{GHz}$ left-hand and right-hand $V$ emission, respectively. In the left and right panels we use black and grey HXT contours for the north and south component respectively; in the middle panel the contours of both HXT components are white. In the left panel, the white curve delineates the flaring loop while the thick black curve shows the radio limb.

maps show that radio emission outlines the flaring loops and peaks close to the loop tops (Figure 4). We try to reproduce the observed microwave morphologies and fluxes using a model gyrosynchrotron loop. The only way to reconcile the observed 17 and $34 \mathrm{GHz}$ morphologies and fluxes is to invoke a magnetic field with constant strength along the model loop. The model loops are thin with aspect ratios of about 0.1 . The derived densities of radio-emitting energetic electrons are $1-6 \times 10^{4} \mathrm{~cm}^{-3}$. The models require the presence of high-energy electrons (as high as $5000 \mathrm{keV}$ ) (Kundu et al. 2000c).

\section{References}

Kundu, M. R., White, S. M., Gopalswamy, N., Lim, J. 1994, ApJ (Suppl. Ser.), 90,599

Kundu, M. R., White, S. M., Shibasaki, K., Sakurai, T., Grechnev, V. V. 2000a, ApJ (in press)

Kundu, M. R., White, S. M., Shibasaki, K., Sakurai, T. 2000b, ApJ (in press)

Kundu, M. R., Nindos, A., White, S. M., Grechnev, V. V. 2000c, ApJ (submitted)

Nindos, A., White, S. M., Kundu, M. R., Gary, D. E. 2000, ApJ, 533, 1053

White, S. M. 1994, in High Energy Solar Phenomena (ed. J. Ryan), AIP Conf. Proceedings 294, 199 